\title{
NOVOS MATERIAIS DE CARNIVORA PARA O PLEISTOCENO DO ESTADO DO RIO GRANDE DO SUL, BRASIL
}

\author{
PATRÍCIA HADLER RODRIGUES \\ Museu de Ciências Naturais, FZB/RS, Av. Dr. Salvador França, 1427, 90690-000, Porto Alegre, RS, Brasil. \\ hadlerpa@uol.com.br \\ FRANCISCOJUAN PREVOSTI \\ LATYR, Museo de La Plata, Paseo del Bosque, 1900, La Plata, Argentina.protocyon@hotmail.com \\ JORGE FERIGOLO, ANA MARIA RIBEIRO \\ Museu de Ciências Naturais, FZB/RS, Av. Dr. Salvador França, 1427, 90690-000, Porto Alegre, RS, Brasil. \\ ferigolo@plug-in.com.br,amr@plug-in.com.br
}

\begin{abstract}
RESUMO - São descritos materiais de Carnivora provenientes do Sistema Laguna-Barreira III da porção sul da Planície Costeira do Estado do Rio Grande do Sul, Neo-Pleistoceno. É apresentado novo material pós-craniano de Smilodon populator e Otaria byronia. Dentes isolados de Dusicyon cf. D. avus e cf. Theriodictis representam o primeiro registro da Família Canidae para este Estado.
\end{abstract}

Palavras-chave: Smilodon populator, Otaria byronia, Canidae, Pleistoceno, Rio Grande do Sul, Brasil.

\begin{abstract}
NEW MATERIAL OF CARNIVORA FROM THE PLEISTOCENE OF RIO GRANDE DO SUL STATE, BRAZIL. Carnivora materials coming from the Sistema Laguna-Barreira III, southern portion of the Coastal Plain of Rio Grande do Sul State, Late Pleistocene are decribed. New postcranial remains of Smilodon populator and Otaria byronia are presented. Isolated teeth of Dusicyon cf. D. avus and cf. Theriodictis represent the first Canidae record for this State.
\end{abstract}

Key words: Smilodon populator, Otaria byronia, Canidae, Pleistocene, Rio Grande do Sul, Brazil.

\section{INTRODUÇÃO}

Os primeiros registros de carnívoros "fissipédios" na América do Sul são do Mioceno superior (Huayqueriense) representados por restos de Procyonidae (Berta \& Marshall, 1978; Webb, 1985; Bond, 1986) e os de pinipédios do Mioceno médio (Cozzuol, 2001). A partir do Plioceno (Marplatense), são encontrados representantes dos Canidae e Mustelidae (Berta \& Marshall, 1978; Webb, 1985; Cione \& Tonni, 1995); e a partir do Ensenadense, os Felidae e Ursidae (Berman,1994; Cione \& Tonni, 1995).

Nos depósitos do Pleistoceno no Rio Grande do Sul, o registro de carnívoros "fissipédios" e pinipédios é escasso. Para os primeiros, até agora é conhecido apenas um representante dos Felidae, Smilodon populator (Moreira, 1970; Bombin, 1975; Oliveira, 1992). Recentemente, foi registrado material de Ursidae Tremarctinae, proveniente do Passo Juquerí, Município de Uruguaiana (Ribeiro et al., 2003).
Pinipédios foram registrados para o Quaternário do Rio Grande do Sul com base em material de Arctocephalus, procedente de Santa Vitória do Palmar (Oliveira \& Drehmer, 1997), e de Otariidae (cf. Otaria byronia), proveniente do município de Mostardas (Drehmer \& Ribeiro, 1998). Dá-se aqui a conhecer novos materiais de Smilodon e Otaria, além de registrar pela primeira vez a Família Canidae para o Estado do Rio Grande do Sul.

\section{LOCALIZAÇÃO E GEOLOGIA}

A Planície Costeira do Rio Grande do Sul (PCRS) é uma ampla área de terras baixas, localizada no leste do Estado (Figura 1), em sua maior parte constituída por um enorme sistema de lagos e lagunas costeiras. Estende-se por $620 \mathrm{~km}$ desde o município de Torres, ao norte, até a desembocadura do arroio Chuí, ao sul (Tomazelli \& Villwock, 1996).

Estratigraficamente, são reconhecidos cinco sistemas 


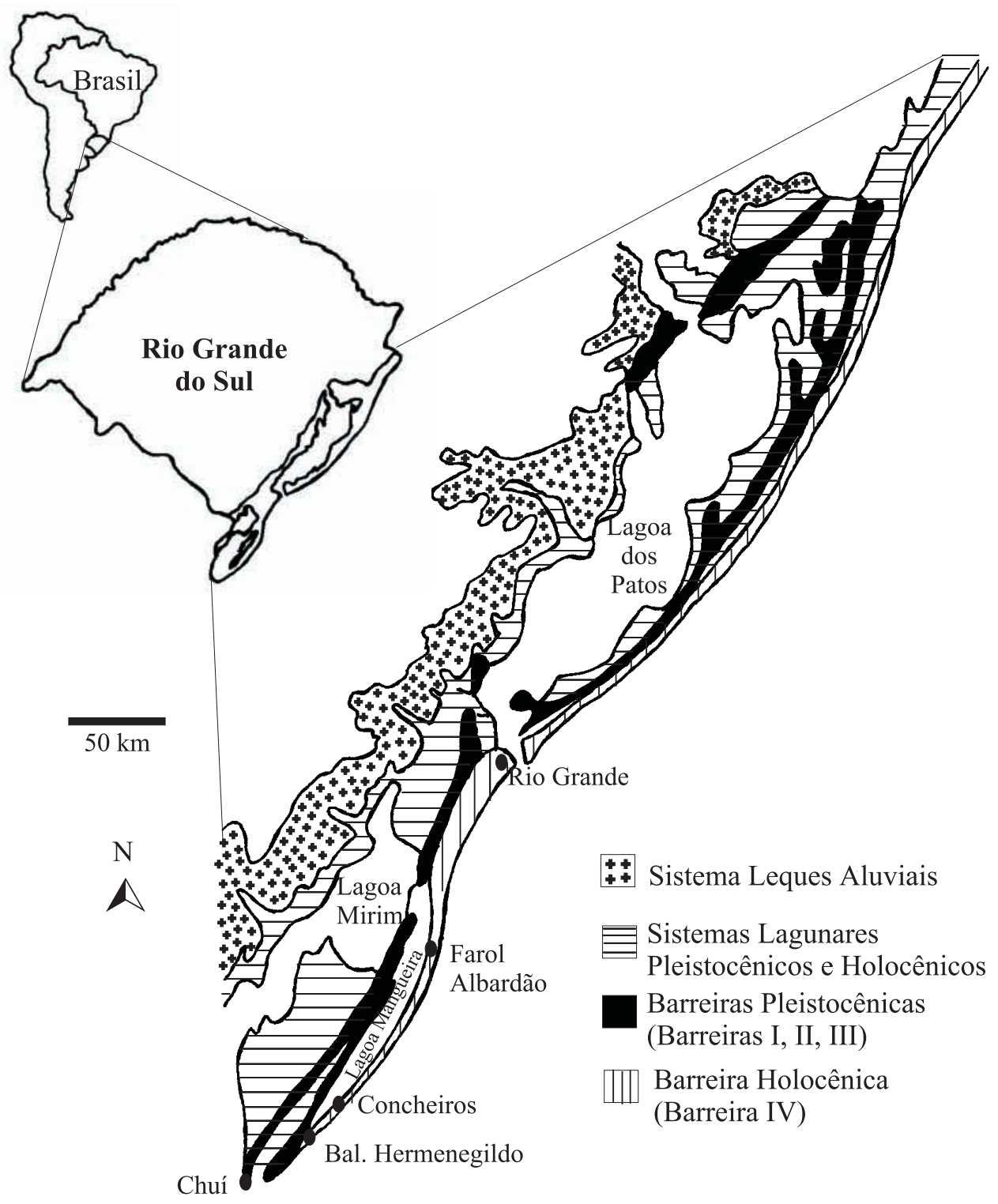

Figura 1. Planície Costeira do RS e sistemas deposicionais (modificado de Tomazelli \& Villwock, 2000).

Figure 1. Coastal Plain of RS and deposicional sequences (modified from Tomazelli \& Villwock, 2000).

deposicionais representados por leques aluviais e quatro sistemas laguna-barreira (SLB; Villwock \& Tomazelli, 1995) I, II, III, IV (Figura 1). O SLB III está relacionado ao último evento transgressivo-regressivo do Pleistoceno (120 ka) e associados a ele ocorrem restos de mamíferos fósseis (Villwock \& Tomazelli, 1995; Buchmann, 2002).

Segundo Ribeiro et al. (1998) e Buchmann (2002), o único afloramento na PCRS com estratigrafia bem conhecida é o do arroio Chuí, onde são encontrados mamíferos terrestres típicos da fauna Lujanense (sensu Cione \& Tonni, 1999), com idade estimada em torno de $120 \mathrm{ka}$ (Buchmann, 2002). Na plataforma continental interna, existem depósitos biodetríticos pleistocênicos, de onde provêm mamíferos (terrestres e marinhos), que são, eventualmente, trazidos à tona pelas redes de pesca. Uma grande quantidade de fósseis também é encontrada na linha de praia atual, como resultado do retrabalhamento de depósitos lagunares pleistocênicos da plataforma continental adjacente (Buchmann \& Rincón-Filho, 1997; Ribeiro et al., 1998). Neste caso, são encontrados, tanto mamíferos terrestres quanto marinhos, sendo os primeiros muito semelhantes taxonomicamente (e.g. Pampatherium, Panochthus, Doedicurus, Glyptodon, Glossotherium, Toxodon, Stegomastodon, Morenelaphus, Blastocerus, Ozotocerus) àqueles do arroio Chuí, sugerindo, assim, idade-mamífero Lujanense. No entanto, a ausência de um contexto estratigráfico preciso impede a determinação acurada da idade deste material. 
Neste trabalho, enfoca-se apenas material coletado na linha de praia da porção sul da PCRS, entre os municípios de Rio Grande e Chuí (Figura 1).

\section{MATERIAL E MÉTODOS}

O material estudado está depositado na Coleção de Paleovertebrados do Museu de Ciências Naturais da Fundação Zoobotânica do Rio Grande do Sul (MCN-PV). O material está constituído por ossos e dentes isolados, coletados ao longo da atual linha de praia. No estudo, o material foi comparado com espécimes fósseis e recentes do Museo Argentino de Ciencias Naturales "Bernardino Rivadavia" (MACN), Museo de La Plata (MLP), Museo de Ciencias Naturales de Mar del Plata "Lorenzo Scaglia" (MMP), Museum National d'Histoire Naturelle, Paris (MNHN), Museo de Ciencias Naturales de Necochea (MCNN), Academy of Natural Sciences of Philadelphia (ANSP), Instituto de la Patagonia, Punta Arenas (CZIP) e Museu de Ciências Naturais da Fundação Zoobotânica do Rio Grande do Sul (MCN-M).

Na nomenclatura dentária seguiu-se Paula-Couto (1979) e, na óssea, a Nômina Anatômica Veterinária (1984), sempre que possível. Na sistemática, seguiu-se McKenna \& Bell (1997), sempre que possível.

\section{SISTEMÁTICA PALEONTOLÓGICA}

Ordem CARNIVORA Bowdich, 1821

Subordem CANIFORMIA Kretzoi, 1943

Família CANIDAE Fischer, 1817

Subfamília CANINAE Fischer, 1817

Gênero Dusicyon Hamilton-Smith, 1839

Dusicyon cf. D. avus Burmeister, 1864

(Figura 2A, 3)
Material.MCN-PV 3514, primeiro molar superior (M1) esquerdo. Procedência. "Concheiros", Município de Santa Vitória do Palmar (Figura 1).

Descrição. Dente com desgaste oclusal acentuado e também bastante rolado. Ele apresenta duas raízes bucais e uma lingual, todas fraturadas, estando a lingual um pouco melhor preservada. O protocone está bem desgastado, localizado mésio-lingualmente e alinhado ao paracone, sendo este maior do que o metacone, além de mais projetado bucalmente. A bacia do trígono é muito bem delimitada pelas cúspides e bastante profunda. $\mathrm{O}$ paracônulo está bem marcado, embora muito desgastado, enquanto o metacônulo é muito discreto, sua presença evidenciada principalmente por uma crista que o une ao protocone e outra ao metacone. O tálon é muito desenvolvido e o hipocone, se presente, está muito desgastado. A bacia do tálon é quase tão profunda quanto aquela do trígono. Um cíngulo possivelmente circundava todo o dente, no entanto, está muito gasto pelo transporte.

Discussão. Este espécime assemelha-se notavelmente, em tamanho e morfologia, a três espécies de canídeos de médio porte: Pseudalopex culpaeus, Dusicyon australis e D. avus. Entre as três espécies, mostra maior semelhança com $P$. culpaeus e D. avus. O metacone e paracone de D. australis é mais agudo e alto, e o cíngulo labial está mais elevado e expandido na sua porção mesial. As dimensões de MCN-PV 3514 (comprimento $=11,7 \mathrm{~mm}$; largura $=18,2 \mathrm{~mm}$ ) são maiores do que as de P. culpaeus e se encontram dentro dos limites de variação de $D$. avus (ver Caviglia, 1986; Berman \& Tonni, 1987; Trejo \& Jackson, 1998; Figura 3). Dusicyon avus é relativamente abundante no Bonaerense-Lujanense da região Pampeana (Berman \& Tonni, 1987; Berman, 1994), ainda que seus primeiros registros sejam do Ensenadense (Prevosti, observação pesssoal). Durante o Pleistoceno tardio-Holoceno, o táxon encontra-se amplamente distribuído na Patagônia e na região Pampeana (Caviglia, 1986; Berman \& Tonni, 1987; Trejo \& Jackson, 1998). Se confirmado, o espécime MCN-PV 3514 é
A

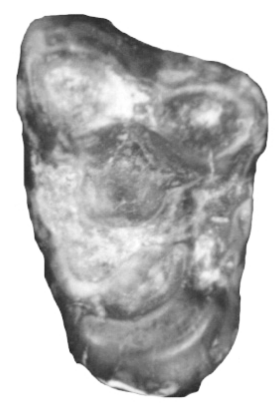

B

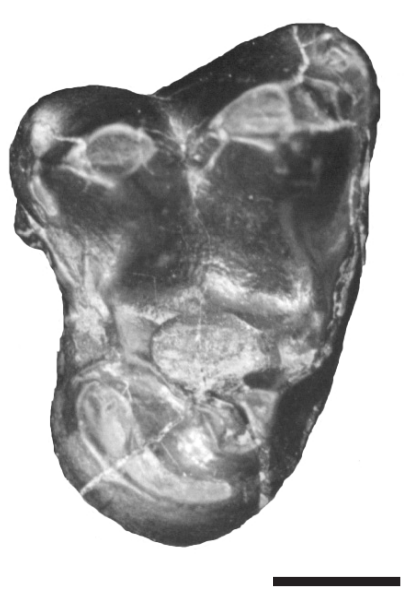

Figura 2. A. Dusicyon cf. D. avus, MCN-PV 3514, M1 esquerdo, vista oclusal; B. cf. Theriodictis, MCN-PV 3639, M1 direito, vista oclusal. Escala $=5 \mathrm{~mm}$.

Figure 2. A. Dusicyon cf. D. avus, MCN-PV 3514, left M1, occlusal view; B. cf. Theriodictis, MCN-PV 3639, right M1, occlusal view. Scale bar $=5 \mathrm{~mm}$ 
o primeiro registro de $D$. avus para o Brasil e também o mais setentrional para a espécie.

\section{Caninae cf. Theriodictis Mercerat, 1891}

(Figura 2B, 4)

Material. MCN-PV 3639, primeiro molar superior (M1) direito. Procedência. Balneário Hermenegildo, Município de Santa Vitória do Palmar (Figura 1).

Descrição. O dente apresenta pouco desgaste, está bem preservado, ainda que rolado; quanto às raízes, uma das bucais está parcialmente preservada, enquanto a outra bucal e a lingual, muito mais robusta do que as demais, estão totalmente preservadas. O protocone é bem diferenciado e menos desenvolvido que o meta- e o paracone, sendo este mais desenvolvido do que o metacone e ligeiramente mais bucal em sua base, ainda que em vista oclusal as cúspides estejam alinhadas. Uma tênue crista projeta-se lingualmente do paracone para unir-se com o protocone. Uma outra crista, menos marcada ainda, une o protocone a um pequeno metacônulo. A bacia do trígono é apenas ligeiramente escavada. O tálon é bem desenvolvido, não havendo todavia um hipocone diferenciado. Cíngulos mesial e distal são estreitos. Mésio-bucalmente, observa-se um pequeno parástilo.

Discussão. MCN-PV 3639 (comprimento $=16,8 \mathrm{~mm}$; largura $=$ $24,1 \mathrm{~mm}$ ) foi comparado aos canídeos de grande porte do

Tabela 1. Medidas ( $\mathrm{mm}$ ) dos úmeros de Otariidae; Otariidae indet. (MCN-PV 3638); Otaria byronia (MCN-PV 2515, MCN-M 2459, 2462, 2505, 2807); Arctocephalus tropicalis (MCN-M 2458, 2463, 2504); A. australis (MCN-M 2461, 2706). Abreviaturas: CT, distância entre a porção mais proximal da cabeça e o sulco da tróclea; ET, espessura da tróclea; DD, menor diâmetro da diáfise.

Table 1. Measurements $(\mathrm{mm})$ of Otariidae humeri; Otariidae indet. (MCN-PV 3638); Otaria byronia (MCN-PV 2515, MCN-M 2459, 2462, 2505, 2807); Arctocephalus tropicalis (MCN-M 2458, 2463, 2504); A. australis (MCN-M 2461, 2706). Abbreviations: CT, distance between the proximal portion of the head and the troclear notch; ET, troclear thickness; DD, lesser diameter of diaphysis.

\begin{tabular}{l|ccc}
\hline & CT & ET & DD \\
\hline MCN-PV 2515 & 215,0 & 38,0 & 45,9 \\
MCN-PV 3638 & 154,1 & 20,1 & 27,1 \\
MCN-M 2459 esq. ? & 208,0 & 28,8 & 44,1 \\
MCN-M 2807 esq. ? & 214,4 & 32,6 & 44,9 \\
MCN-M 2807 dir. ? & 216,7 & 35,5 & 45,1 \\
MCN-M 2505 esq. ? & - & 35,2 & 44,4 \\
MCN-M 2504 esq. ? & 150,0 & 20,5 & 25,8 \\
MCN-M 2504 dir. ? & 151,7 & 19,7 & 26,1 \\
MCN-M 2458 esq. ? & 156,6 & 18,8 & 25,6 \\
MCN-M 2458 dir. ? & 155,6 & 18,7 & 26,3 \\
MCN-M 2463 esq. ? & 157,1 & 21,4 & 32,4 \\
MCN-M 2463 dir. ? & 156,6 & 20,7 & 32,7 \\
MCN-M 2461 esq. ? & 151,5 & 25,5 & 30,7 \\
MCN-M 2461 dir. ? & 156,6 & 27,0 & 35,0 \\
MCN-M 2706 esq. ? & 155,1 & 19,8 & 25,5 \\
MCN-M 2706 dir. ? & 155,0 & 19,4 & 26,1 \\
MCN-M 2462 esq. ? & 157,0 & 21,9 & 27,0 \\
MCN-M 2462 dir. ? & 156,3 & 21,9 & 26,9 \\
\hline
\end{tabular}

Tabela 2. Medidas ( $\mathrm{mm}$ ) do metacarpal V de Smilodon populator. Table 2. Measurements $(\mathrm{mm})$ of metacarpal $\mathrm{V}$ of Smilodon populator.

\begin{tabular}{l|c}
\hline MCN -PV \\
$\mathbf{3 6 3 1}$
\end{tabular}

Pleistoceno: Protocyon, Speothos, Theriodictis, Chrysocyon e Canis. Além de ser menor, Protocyon difere do novo material por não apresentar tálon e nem metacônulo, (Winge, 18951896, plate II, fig. 1A; Berta, 1987). Speothos, também é menor, e possui uma ampla bacia do trígono e um grande metacônulo unido ao protocone, que é baixo e pequeno (Berta, 1984). Chrysocyon difere quanto à morfologia, pois este apresenta grande metacônulo e também metástilo (Pereira, 1977). O tamanho e a redução do cíngulo labial o aproxima de Theriodictis e Canis gezi. Nas demais espécies sul-americanas de Canis, este cíngulo está bem desenvolvido, à exceção de alguns exemplares de $C$. dirus, nos quais está pouco marcado no limite metacone-paracone (Berta, 1988). O M1 de C. gezi possui um metacônulo maior e é proporcionalmente mais largo. Contrariamente, em Theriodictis, o hipocone do M1 está mais reduzido. É importante ressaltar que Prevosti (2001) apontou vários caracteres em comum entre Theriodictis e o holótipo de $C$. gezi, sugerindo que este poderia ser um sinônimo de $T$. platensis. Caso confirme-se esta hipótese, as diferenças observadas na morfologia de M1 (grau de desenvolvimento do hipocone e do metacônulo) seriam consideradas apenas como variação intraespecífica. Neste sentido, a morfologia do espé-

Tabela 3. Medidas $(\mathrm{mm})$ das falanges proximais do dígito I, II e V da mão de Smilodon populator.

Table 3. Measurements (mm) of proximal phalanx, digit I, II and $\mathrm{V}$, manus of Smilodon populator.

\begin{tabular}{l|ccccc}
\hline & MCN-PV & MCN-PV & MCN-PV & MCN-PV & MCN- PV \\
& $\mathbf{3 6 3 4}$ & $\mathbf{3 6 3 5}$ & $\mathbf{3 6 3 6}$ & $\mathbf{3 6 3 7}$ & $\mathbf{3 6 3 0}$ \\
\hline $\begin{array}{l}\text { Maior } \\
\text { comprimento }\end{array}$ & 43,1 & 50,5 & 37,6 & 43,2 & 36,5 \\
$\begin{array}{l}\text { Maior largura } \\
\text { na porção } \\
\text { proximal }\end{array}$ & 21,5 & 21,6 & 16,1 & 21,2 & 24,7 \\
$\begin{array}{l}\text { Largura no } \\
\text { centro do corpo }\end{array}$ & 15,4 & 15,0 & 12,9 & 14,5 & 21,6 \\
$\begin{array}{l}\text { Maior largura } \\
\text { na porção } \\
\text { distal }\end{array}$ & 15,2 & 15,1 & 12,5 & 16,9 & 22,5 \\
\hline
\end{tabular}


Tabela 4. Medidas ( $\mathrm{mm}$ ) da falange média do dígito III da mão de Smilodon populator.

Table 4. Measurements ( $\mathrm{mm}$ ) of middle phalanx, digit III, manus of Smilodon populator.

\begin{tabular}{l|c}
\hline & $\begin{array}{c}\text { MCN- PV } \\
\mathbf{1 6 4 8}\end{array}$ \\
\hline Maior comprimento & 33,3 \\
$\begin{array}{l}\text { Maior largura na } \\
\text { porção proximal }\end{array}$ & 20,3 \\
$\begin{array}{l}\text { Largura no centro do } \\
\text { corpo }\end{array}$ & 14,8 \\
$\begin{array}{l}\text { Maior largura na } \\
\text { porção distal }\end{array}$ & 20,3 \\
\hline
\end{tabular}

cime MCN-PV 3639 encontra-se dentro de tal limite de variação. Este é o primeiro registro do gênero para o Brasil. Os registros prévios de Theriodictis são para a Argentina e a Bolívia, em níveis de idade-mamífero Ensenadense (ver Prevosti et al., no prelo). A escassez de material, a ausência de dados estratigráficos e o fato de o material ser transportado impede uma atribuição precisa quanto à sua idade.

\author{
Família OTARIIDAE Gill, 1866 \\ Gênero Otaria Peron, 1816 \\ Otaria byronia (Blainville, 1820) \\ (Figura 5, 6 A-B, Tabela 1)
}

Material. MCN-PV 2515, úmero esquerdo incompleto. Procedência. Farol do Albardão, Município de Santa Vitória do Palmar (Figura 1).

Descrição. Úmero esquerdo faltando parte da cabeça, de ambos tubérculos, toda a crista deltóide, pequena parte dos epicôndilos e das margens da tróclea. O úmero é robusto e a porção preservada do tubérculo maior projeta-se bastante proximalmente à cabeça. O tubérculo menor, cuja extremidade não atinge o nível da cabeça, se prolonga distalmente quase até o nível da tuberosidade deltóide. Uma tênue crista inicia-se na face posterior deste tubérculo, projetando-se distal e lateralmente até o início da "metáfise" de onde por curta distância se estende medialmente, até quase atingir a fossa olecraniana. $\mathrm{O}$ sulco intertubercular é amplo e a crista deltóide se prolonga distalmente até a discreta fossa coronóide. A fossa olecraniana, mais ou menos triangular, está bastante marcada. O epicôndilo lateral, embora fraturado, era mais delicado do que o medial e se projetava algo posteriormente. O medial é mais distal e mais desenvolvido que o lateral. $\mathrm{O}$ forame entepicondilar está ausente. Toda a margem da superfície articular distal é bastante proeminente anterior, medial e lateralmente, embora seja mais discreta posteriormente. O capítulo tem forma semi-esférica, com margem lateral ligeiramente convexa. A porção coronóide da tróclea é mais profunda e menos convexa do que a porção olecraniana.

Discussão. Na morfologia, mas principalmente no tamanho, este úmero assemelha-se muito com o de espécimes recentes de machos de Otaria byronia (e.g. MCN-M 2459, 2505, 2807). Fêmeas de $O$. byronia e fêmeas e machos de Arctocephalus
Tabela 5. Medidas $(\mathrm{mm})$ dos astrágalos de Smilodon populator. Table 5. Measurements $(\mathrm{mm})$ of Smilodon populator astragali

\begin{tabular}{l|cc}
\hline & $\begin{array}{c}\text { MCN-PV } \\
\mathbf{3 6 3 2}\end{array}$ & $\begin{array}{c}\text { MCN-PV } \\
\mathbf{3 6 3 3}\end{array}$ \\
\hline $\begin{array}{l}\text { Maior } \\
\text { comprimento }\end{array}$ & 55,3 & 50,7 \\
Maior largura & 55,6 & 50,6 \\
$\begin{array}{l}\text { Maior diâmetro da } \\
\text { cabeça }\end{array}$ & 36,2 & 30,1 \\
\hline
\end{tabular}

apresentam úmeros com dimensões muito inferiores (ver Tabela 1, Figura 5).

OTARIIDAE indet.

(Figura 5, 6 C-D; Tabela 1)

Material. MCN-PV 3638, úmero esquerdo incompleto.

Procedência. Balneário Hermenegildo, Município de Santa Vitória do Palmar (Figura 1).

Descrição. Úmero esquerdo bastante fraturado e alterado pelo intemperismo, faltando a margem da cabeça, todo o grande tubérculo, parte da crista deltóide e do pequeno tubérculo. Este espécime distingue-se do anterior (MCN-PV 2515) pelo menor tamanho e robustez, pelo epicôndilo medial proporcionalmente maior e o lateral menos inclinado posteriormente, pela fossa do olécrano proporcionalmente menos profunda, e pelas bordas da tróclea e do capítulo não tão bem marcadas. Discussão. Nas dimensões e na morfologia o espécime é compatível tanto com os espécimes machos de Arctocephalus quanto com os de fêmeas de Otaria byronia (ver Tabela 1), o que não permite atribuição abaixo do nível de família.

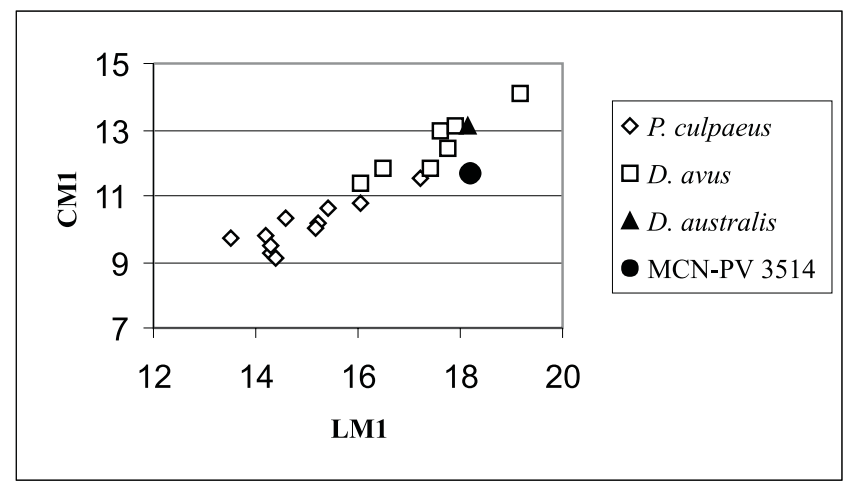

Figura 3. Gráfico bivariado comparando o comprimento (CM1; mm) vs. a largura (LM1; mm) do M1 de Dusicyon cf. D. avus (MCN-PV 3514) com espécimes de D. avus (MLP 81-VI29-2, MLP 96-V-2-1, MLP 10-60, MLP 10-53, MLP 306, MLP 307, MACN 53), D. australis (ANSP 588) e Pseudalopex culpaeus (MLPMa 1264, MLPMa 27-X97-29, MLPMa 1265, MLPMa 5-III-36-10, MLPMa 1749, MLPMa $27-$ X-97-10, MLPMa 1267, MLPMa 5-III-36-9, MLPMa 1266, MLPMa 5III-36-21, CZIP 0218).

Figure 3. Bivariate graphic comparing $\mathrm{M} 1$ length $(\mathrm{CM} 1 ; \mathrm{mm})$ vs width (LM1; mm) of Dusicyon cf. D. avus (MCN-PV 3514) with D. avus (MLP 81-VI29-2, MLP 96-V-2-1, MLP 10-60, MLP 10-53, MLP 306, MLP 307, MACN 53), D. australis (ANSP 588) and Pseudalopex culpaeus (MLPMa 1264, MLPMa 27-X-97-29, MLPMa 1265, MLPMa 5-III-36-10, MLPMa 1749, MLPMa 27-X-97-10, MLPMa 1267, MLPMa 5-III-36-9, MLPMa 1266, MLPMa 5-III-36-21, CZIP 0218) specimens. 


\author{
Subordem FELIFORMIA Kretzoi, 1945 \\ Família FELIDAE Fischer, 1817 \\ Subfamília MACHAIRODONTINAE Gill, 1872 \\ Gênero Smilodon Lund, 1842 \\ Smilodon populator Lund, 1842
}

(Figura 7; Tabelas 2-5)

Material. MCN-PV 3631, metacarpal V direito; MCN-PV 3630, falange proximal do dígito I da mão esquerda; MCN-PV 3634, $3635,3636,3637$, falanges proximais dos dígitos II ou V da mão direita; MCN-PV 1648, falange média do dígito III da mão direita; MCN-PV 3632, 3633, astrágalos direitos.

Procedência. Balneário Hermenegildo, Município de Santa Vitória do Palmar (Figura 1).

\section{Descrição e discussão}

Metacarpal V direito. MCN-PV 3631 (Figura 7 A-C; Tabela 2) assemelha-se ao metacarpal V descrito por Merriam \& Stock (1932) e Paula-Couto (1980). O corpo apresenta uma pequena tuberosidade fusiforme na porção medial da face palmar, e sua secção transversal é triangular. Entre a cabeça e o corpo há um profundo sulco em forma de "V", de vértice posterior, no ápice do qual há uma fossa com um grande forame vascular. Lateralmente ao "V" há, de cada lado, um processo proeminente que se projeta para além dos limites da cabeça. O limite medial da cabeça é mais ou menos plano e de forma semilunar; sendo o lateral côncavo e ligeiramente oblíquo.

Falange proximal do dígito I da mão esquerda. MCN-PV 3630 (Figura 7 D,E; Tabela 3) assemelha-se àquele descrito por Merriam \& Stock (1932), Paula-Couto (1980) e Mello (1989) em relação aos caracteres da base e da cabeça. O corpo é curto e robusto, e ventralmente há uma fossa que se limita com a cabeça por um sulco em forma de "V", de vértice distal.

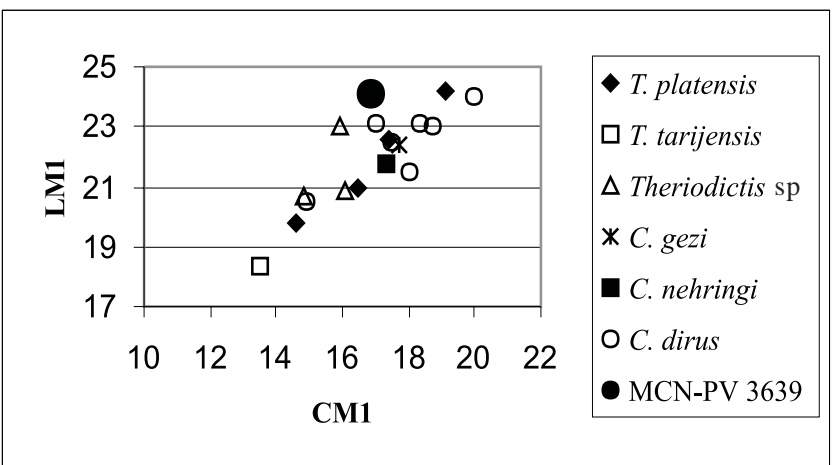

Figura 4. Gráfico bivariado comparando o comprimento (CM1; $\mathrm{mm})$ vs. a largura (LM1; mm) do M1 de cf. Theriodictis (MCN-PV 3639) com espécimes de T. platensis (MLP 10-51, MLP 96-IX-11, MMP 2700, MCNN 941), T. tarijensis (MACN1452), Theriodictis sp.(MACN 11606, MACN 11611, MNHN TAR 662), Canis gezi (MACN 5120), C. nehringi (MACN500) e C. dirus (Merriam, 1912; Berta, 1988).

Figure 4. Bivariate graphic comparing $\mathrm{M} 1$ length $(\mathrm{CM} 1 ; \mathrm{mm})$ vs. width (LM1; mm) of cf. Theriodictis (MCN-PV 3639) with T. Platensis (MLP 10-51, MLP 96-IX-1-1, MMP 2700, MCNN 941), T. Tarijensis (MACN1452), Theriodictis sp. (MACN 11606, MACN 11611, MNHN TAR 662), Canis gezi (MACN 5120), C. nehringi (MACN500) and $C$. dirus (Merriam, 1912; Berta,1988) specimens.
Na face lateral de cada lado da cabeça, mais dorsalmente, há uma fosseta para inserção de ligamento.

Falanges proximais (dígitos II ou V ?) da mão direita. MCNPV 3634-3637 (Figura 7 F, G; Tabela 3) é semelhante às falanges proximais descritas por Paula-Couto (1980) e Mello (1989). Ventralmente à faceta para o metacarpal, há uma fossa. Na porção distal do corpo há, de cada lado, uma pequena projeção para inserção de tendão muscular. De cada lado, nas faces laterais da cabeça, há uma fosseta para inserção de ligamentos laterais. O espécime MCN-PV 3637 apresenta na face medial, uma concavidade bem marcada.

Falange média do dígito III da mão direita. Na região proximal de MCN-PV 1648 (Figura 7 H, I; Tabela 4), falta a porção entre as duas facetas articulares, de modo que não há a "pseudo extraarticular facet" descrita por Paula-Couto (1980:199). Nesta região Merriam \& Stock (1932: 162, fig. 131) ilustram um pequeno processo, mas não se referem a uma faceta articular extra. A fossa ventral às facetas articulares proximais é mais profunda do que nas falanges proximais (MCN-PV 3634, 3635, 3636, 3637). O corpo é mais curto que nas proximais, com a face ventral plana e a dorsal com uma crista arredondada que divide a mesma em uma porção medial plana e numa lateral marcadamente côncava. As fossetas ligamentares laterais à cabeça são menos profundas que nas falanges proximais (MCN-PV 3630, 3634, $3635,3636,3637)$.

Astrágalos direitos. Os espécimes MCN-PV 3632 e MCN-PV 3633 apresentam certas particularidades (Figura 7 J-M; Tabela 5) em relação aos descritos por Ameghino (1905a,b), Merriam \& Stock (1932), Mendez-Alzola (1941), Kraglievich (1948) e Mello (1989), principalmente em relação às facetas articulares para o câlcaneo. A faceta ectal é ovalada e menos projetada distalmente no MCN-PV 3632 do que no MCN-PV 3633, onde é retangular distalmente, devido à presença de uma projeção semilunar ântero-

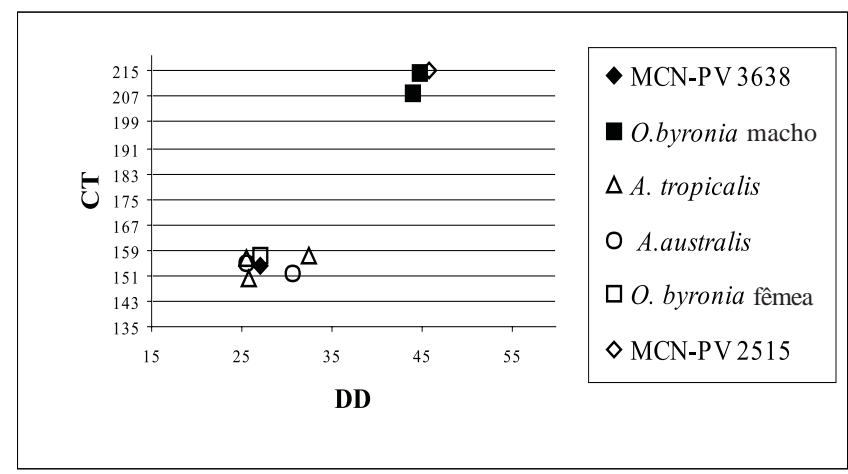

Figura 5. Gráfico bivariado comparando o menor diâmetro da diáfise (DD; mm) vs. a distância entre a porção mais proximal da cabeça e o sulco da tróclea (CT; $\mathrm{mm}$ ) do úmero esquerdo dos espécimes MCN-PV 2515, 3638 com espécimes de Otaria byronia macho (MCN-M 2459, 2807) e fêmea (MCN-M 2462) e Arctocephalus australis (MCN-M 2461, 2706) e A. tropicalis (MCN-M 2458, 2463, 2504) machos.

Figure 5. Bivariate graphic comparing less diameter of diaphysis (DD; $\mathrm{mm}$ ) vs. distance between the proximal portion of the head and the troclear notch (CT; mm) of MCN-PV 2515, 3638 with males (MCN-M 2459, 2807) and female (MCN-M 2462) of Otaria byronia and males of Arctocephalus australis (MCN-M 2461, 2706) e $A$. tropicalis (MCN-M 2458, 2463, 2504). 

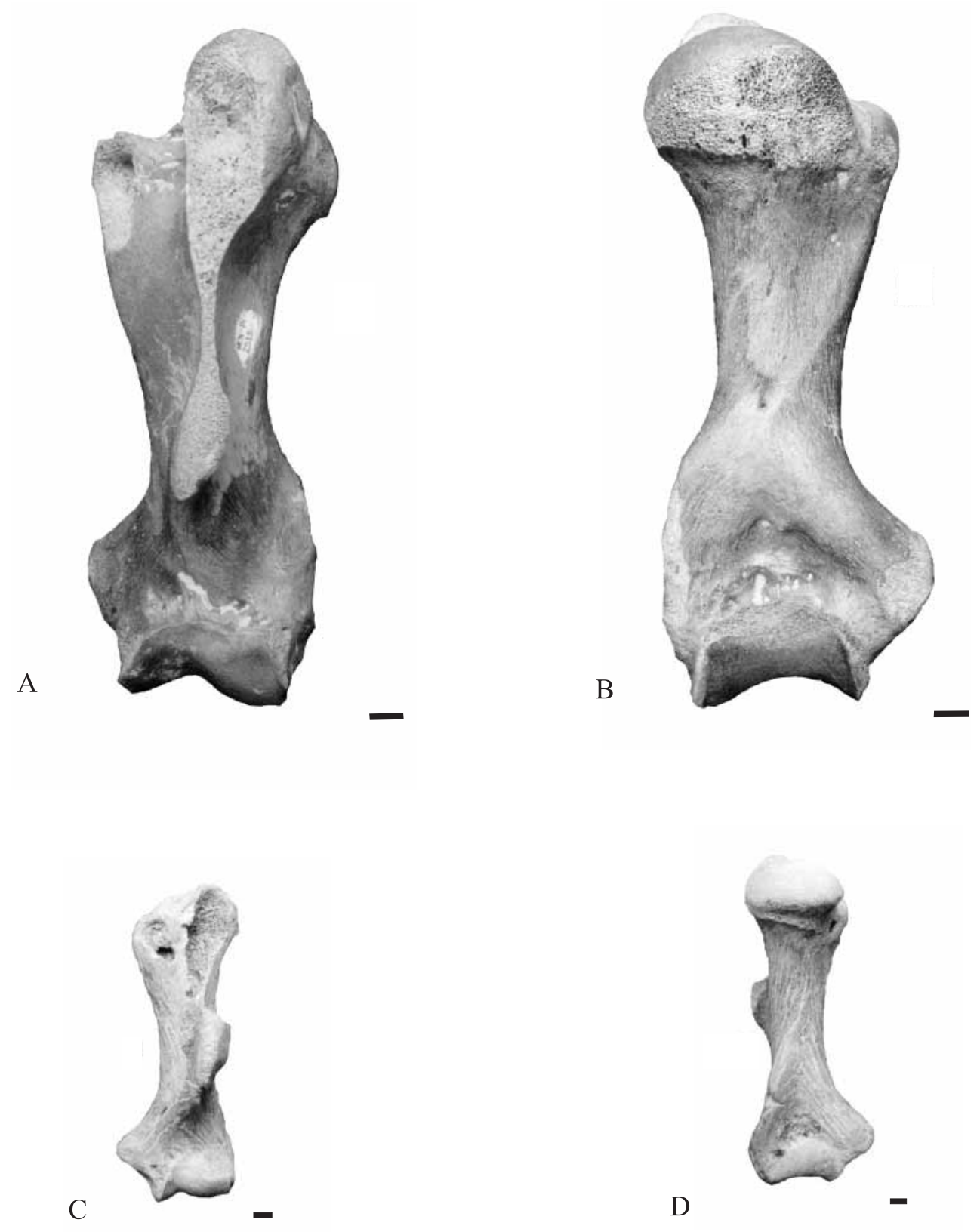

Figura 6. A, B. Otaria byronia, MCN-PV 2515, úmero esquerdo; C, D. Otariidae indet., MCN-PV 3638, úmero esquerdo; A, C. vista anterior; B, D. vista posterior. Escala $=10 \mathrm{~mm}$.

Figure 6. A, B. Otaria byronia, MCN-PV 2515, left humerus; C, D. Otariidae indet., MCN-PV 3638, left humerus; A, C. anterior view; B, D. posterior view; Scale bar $=10 \mathrm{~mm}$

medial. A faceta sustentacular no espécime MCN-PV 3633 apresenta uma porção vertical, que quase alcança a margem da ponte, a qual está ausente no MCN-PV 3632. Embora parcialmente fraturada em ambos espécimes, há uma união da faceta sustentacular com a terceira faceta (vide Merriam \& Stock, 1932) para o calcâneo, planto-lateralmente situada à cabeça, faceta esta discretamente convexa. A faceta fibular é mais profunda no MCN-PV 3632. A ponte óssea está unida medialmente à tróclea apenas em MCN-PV 3633. O forame astragalar é conspícuo nos dois espécimes. As diferenças morfológicas e de tamanho entre os espécimes poderiam dever-se tanto a diferentes faixas etárias quanto a variação anatômica.

\section{CONSIDERAÇÕES FINAIS}

São registrados novos materiais de carnívoros para o Pleistoceno do Rio Grande do Sul. O material adicional de mão e pé de S. populator contribui para o conhecimento da variabilidade intraespecífica da forma brasileira dessa espécie, visto que esta apresenta uma ampla distribuição.

O novo material de Otariidae constitui-se no terceiro registro fóssil dessa família para o Estado do Rio Grande do Sul e o primeiro de material pós-craniano para Otaria byronia. Maiores estudos da fauna "aquática", principalmente dos mamíferos, serão necessários para que futura- 


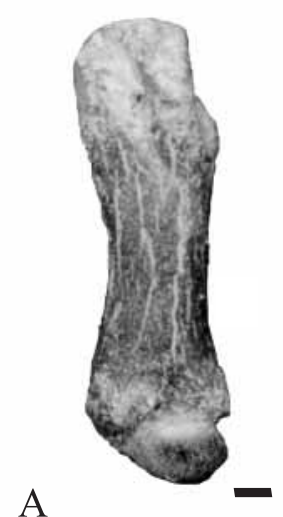

A

B

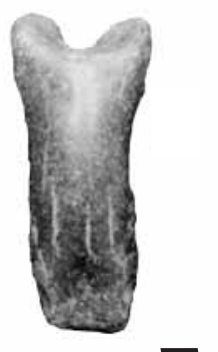

$$
\text { F }
$$

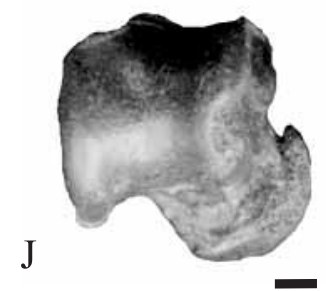

G

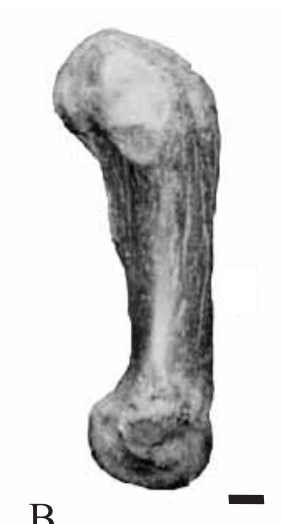

C

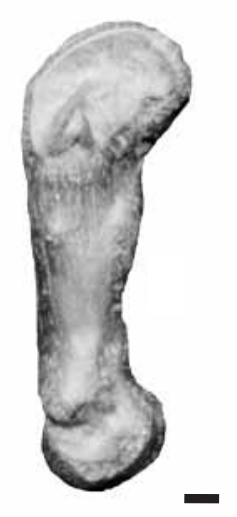

$\mathrm{D}$

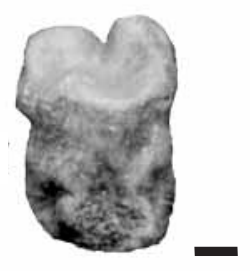

$\mathrm{E}$

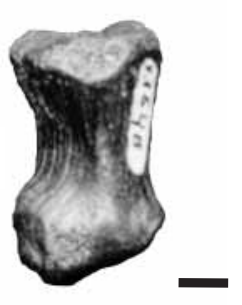

$\mathrm{H}$
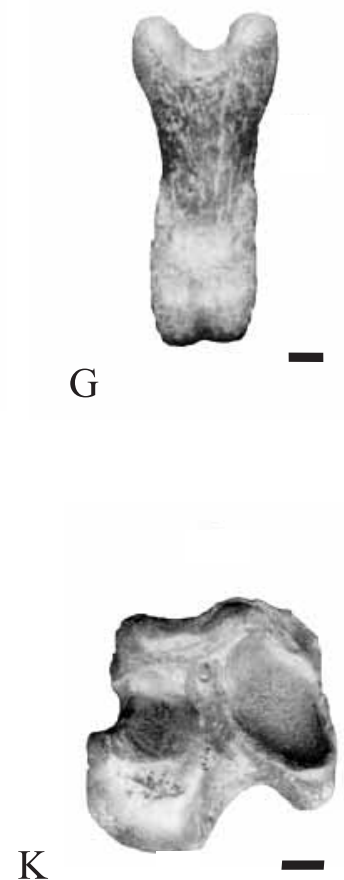

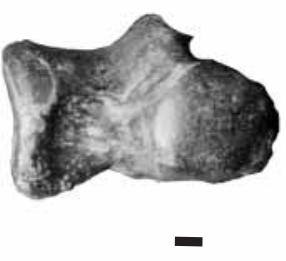

$\mathrm{L}$
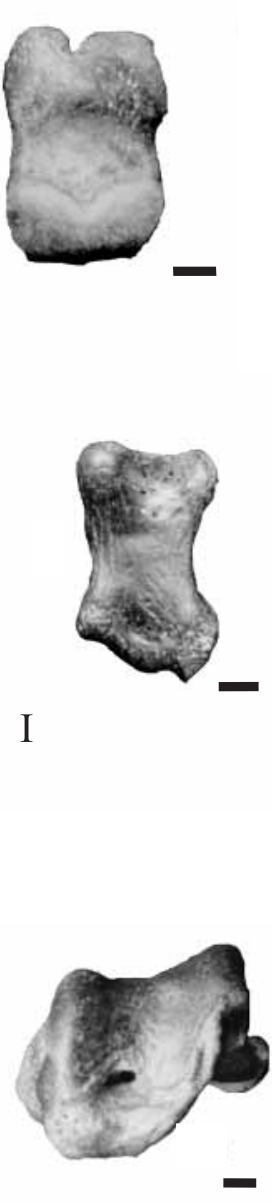

M

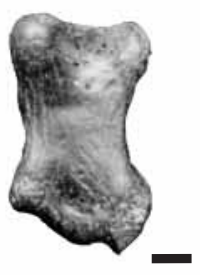

Figura 7. Smilodon populator; A-C. MCN-PV 3631, metacarpal V direito, A, Vista dorsal; B, Vista lateral; C, Vista medial; D-E. MCN-PV 3630, falange proximal do dígito I da mão esquerda; F-G, MCN-PV 3635, falange proximal da mão direita (dígito II ou V?); H-I, MCN-PV 1648, falange média do dígito III da mão direita; D, F, H, Vista dorsal; E, G, I, vista palmar; J-M, MCN-PV 3632, astrágalo direito, J, vista proximal; $\mathbf{K}$, vista plantar; L, vista distal; $\mathbf{M}$, vista posterior. Escala $=5 \mathrm{~mm}$

Figure 7. Smilodon populator; A-C, MCN-PV 3631, right metacarpal V; A, dorsal view; B, lateral view; C, medial view; D-E, MCN-PV 3630, left digit I, manus, proximal phalanx; F-G, MCN-PV 3635, right manus, (digit II or V?) proximal phalanx; H-I, MCN-PV 1648, right digit III, manus, middle phalanx; D, F, H, dorsal view; E, G, I, palmar view; J-M, MCN-PV 3632, right astragalus, J, proximal view; K, plantar view; L, distal view; M, posterior view. Scale bar $=5 \mathrm{~mm}$

mente se possa correlacionar esta com outras faunas sulamericanas.

A Família Canidae é registrada pela primeira vez para o Pleistoceno do Estado do Rio Grande do Sul, representada por dentes isolados de Dusicyon cf. D. avus e cf. Theriodictis, sendo este também o primeiro registro desses táxons para o Brasil. A ocorrência de D. avus no RS vem ampliar a sua área de distribuição no Lujanense, para uma latitude mais ao norte das anteriormente registradas.

\section{AGRADECIMENTOS}

A Francisco Buchmann e Luiz Rota de Oliveira, pela coleta do material estudado. A Alejandro Kramarz (MACN, Buenos 
Aires), Marcio Martins (MCN, Porto Alegre), Christian De Muizon (MNHN, Paris), Alejandro Dondas (MMP, Mar Del Plata), Jaime Carcamo (CZIP, Punta Arenas), Marcelo Reguero (MLP, La Plata) e Mariano Merino (MLP, La Plata) por permitirem o acesso ao material das coleções. PHR e FJP agradecem à CAPES e à Comision de Investigaciones Cientificas (Buenos Aires) pela concessão das bolsas. Aos árbitros anônimos, pelas valiosas sugestões.

\section{REFERÊNCIAS BIBLIOGRÁFICAS}

Ameghino, F. 1905a. La perforación astragaliana en los mamíferos no es un carácter originariamente primitivo. Anales del Museo Nacional de Buenos Aires, 3(4):349-460.

Ameghino, F. 1905b. La faceta articular inferior unica del astragalo de algunos mamíferos, no es un carácter primitivo. Anales del Museo Nacional de Buenos Aires, 3(5):1-64.

Berman, W.D. 1994. Los carnívoros continentales (Mammalia, Carnivora) del Cenozoico en la Provincia de Buenos Aires. Facultad de Ciencias Naturales y Museo, Universidad Nacional de La Plata, Tese de Doutorado, 412 p.

Berman, W.D. \& Tonni, E.P. 1987. Canis (Dusicyon) avus Burmeister, 1864 (Carnivora, Canidae) en el Pleistoceno tardío y Holoceno de la Provincia de Buenos Aires. Aspectos sistemáticos y bioestratigráficos relacionados. Ameghiniana, 24 (3-4):245-250.

Berta, A. 1984. The Pleistocene Bush Dog Speothos pacivorus (Canidae) from the Lagoa Santa caves, Brazil. Journal of Mammalogy., 65(4):549-559.

Berta, A. 1987. Origin, diversification, and zoogeography of the South American Canidae. Fieldiana, Zoology, 39:455-471.

Berta, A. 1988. Quaternary evolution and biogeography of the large South American Canidae (Mammalia: Carnivora). University of California Publications, Geological Sciences, 132:1-149.

Berta, A. \& Marshall, L.G. 1978. South American Carnivora. In: F. Westphal (ed.) Fossilium Catalogus- I: Animalia, W. Junk b.v. Publishers, p.1-47.

Bombin, M. 1975. Afinidade paleoecológica, cronológica e estratigráfica do componente de megamamíferos na biota do Quaternário terminal da Província de Buenos Aires (Argentina), Uruguai e Rio Grande do Sul (Brasil). Comunicações do Museu de Ciências da PUCRS, Zoologia, 9:1-28.

Bond, M. 1986. Los carnívoros terrestres fósiles de Argentina: resumen de su historia. In: CONGRESO ARGENTINO DE PALEONTOLOGÍA Y BIOSTRATIGRAFÍA, 4, 1986. Actas, Mendoza, 2:167-171.

Buchmann, F.S.C. 2002. Bioclastos de organismos terrestres e marinhos na praia e plataforma interna do Rio Grande do Sul: natureza, distribuição, origem e significado geológico. Programa de Pós-Graduação em Geociências, Universidade Federal do Rio Grande do Sul, Tese de Doutorado, 108 p.

Buchmann, F.S.C. \& Rincón-Filho, G. 1997. Fósseis de vertebrados marinhos do Pleistoceno Superior na porção sul da Planície Costeira do Rio Grande do Sul, Brasil. Notas Técnicas, 10:7-16.

Caviglia, S.E. 1986. Nuevos restos de cánidos tempranos en sitios arqueológicos de Fuego-Patagonia. Anales del Instituto de la Patagonia, Serie Ciencias Sociales, 16:85-93.

Cione, A.L. \& Tonni, E.P. 1995. Biostratigrafía y cronología del Cenozoico superior de la Región Pampeana. In: M. T. Alberdi; G. Leone \& E. P. Tonni (eds.) Evolución biológica y climática de la región pampeana durante los últimos cinco millones de años - un ensayo de correlación con el mediterráneo occidental, Monografias del Museo Nacional de Ciencias Naturales, p. 47-74.
Cione, A.L. \& Tonni, E.P. 1999. Biostratigraphy and chronological scale of uppermost Cenozoic in the Pampean area, Argentina. Quaternary of South America and Antartic Peninsula, 12:23-52.

Cozzuol, M. 2001. A "Northern" seal from the Miocene of Argentina: implicatios for phocid phylogeny and biogeography. Journal of Vertebrate Paleontology, 21(3):415-421.

Drehmer, C.J. \& Ribeiro, A.M. 1998. A temporal bone of an Otariidae (Mammalia, Pinnipedia) from the late Pleistocene of Rio Grande do Sul State, Brazil. Revista Universidade Guarulhos, Geociências, 3(6):39-44.

Kraglievich, L.J. 1948. Smilodontidion rigii, n. gen. n. sp. un nuevo y pequeño esmilodonte en la fauna pliocena de chapadmalal. Revista del Museo Argetino de Ciencias Naturales "Bernardino Rivadavia”, 1(1):1- 44.

McKenna, M.C. \& Bell, S.K. 1997. Classification of mammals above the species level. New York, Columbia University Press, $631 \mathrm{p}$.

Mello, M.G. da S. 1989. Mamíferos quaternários de Itapipoca, Ceará, Brasil, depositados no Museu Nacional, Rio de Janeiro. Programa de Pós-Graduação em Zoologia, Museu Nacional, Universidade Federal do Rio de Janeiro, Dissertação de Mestrado, $150 \mathrm{p}$.

Mendez-Alzola, R. 1941. El Smilodon bonaerensis (Muñiz) estudio osteológico y osteométrico del gran tigre fósil de la pampa comparado con otros félidos actuales y fósiles. Anales del Museo Argentino de Ciencias Naturales "Bernardino Rivadavia”, 40 (67):135- 252.

Merriam, J.C. 1912. The fauna of Rancho La Brea. Part II. Canidae. Memoirs of the University of California, 1:216-273.

Merriam, J.C. \& Stock, C. 1932. The felidae of Rancho La Brea. Carnegie Institute Washington, 422:1- 231.

Moreira, L.E. 1970. Primeira ocorrência de Smilodon populator Lund, 1842 no Estado do Rio Grande do Sul. Pesquisas, Zoologia, 23:25-35.

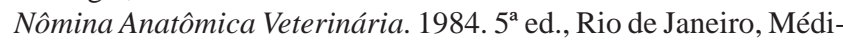
ca e Científica, 110 p.

Oliveira, E.V. 1992. Mamíferos fósseis do Quaternário do Estado do Rio Grande do Sul, Brasil. Programa de Pós-Graduação em Geociências, Universidade Federal do Rio Grande do Sul, Dissertação de Mestrado, 113 p.

Oliveira, E.V. \& Drehmer, C.J. 1997. Sobre alguns restos de Pinnipedia-Otariidae (Mammalia, Carnivora) do Quaternário do Estado do Rio Grande do Sul, Brasil. Revista Universidade Guarulhos, Geociências, 2(6):19-22.

Paula-Couto, C. 1979. Tratado de Paleomastozoologia. Rio de Janeiro, Academia Brasileira de Ciências, 590 p.

Paula-Couto, C. 1980. Pleistocene mammals from Minas Gerais and Bahia, Brazil. In: CONGRESO ARGENTINO DE PALEONTOLOGÍA Y BIOSTRATIGRAFÍA,2, Y CONGRESO LATINO-AMERICANO DE PALEONTOLOGIA, 1, 1989. Acta, Buenos Aires, 3, p.193-209.

Pereira, J.F.P. 1977. Osteologia craniana e odontologia de Chrysocyon brachyurus (Illiger, 1815). Programa de Pós-Graduação em Geociências, Universidade Federal do Rio Grande do Sul, Dissertação de Mestrado, 40 p.

Prevosti, F.J. 2001. The fossil record of Canis (Carnivora: Canidae) in South America. Comments on the systematic status of $\mathrm{Ca}$ nis gezi. In: CANID BIOLOGY AND CONSERVATION CONFERENCE, 2001. Abstracts, Oxford, p. 94.

Prevosti, F.J.; Dondas A. \& Isla F.I. (no prelo). Revisión del registro fósil de Theriodictis Mercerat, 1891 (Carnivora, Canidae) y descripción de un nuevo ejemplar de Theriodictis platensis Mercerat, 1891 del Pleistoceno de la provincia de Buenos Aires (Argentina). Ameghiniana. 
Ribeiro, A.M; Drehmer, C.J.; Buchmann, F.S.C. \& Simões-Lopes, P.C. 1998. Pleistocene skull remains of Pontoporia blainvillei (Cetacea, Pontoporiidae) from the Coast Plain of Rio Grande do Sul State, Brazil, and the relationship of pontoporiids. Revista Universidade Guarulhos, Geociências, 3(6):71-77.

Ribeiro, A.M.; Rodrigues, P.H. \& Ferigolo, J. 2003. Primeiro registro de Ursidae (Tremarctinae) para o Pleistoceno do Estado do Rio Grande do Sul, Brasil. In: JORNADAS ARGENTINAS DE PALEONTOLOGIA DE VERTEBRADOS, 19, 2003. Resúmenes, Buenos Aires, p. 27.

Tomazelli, L.J. \& Villwock, J.A. 1996. Quaternary geological evolution of Rio Grande do Sul Coastal Plain, Southern Brazil. Anais da Academia Brasileira de Ciências, 68(3): 373-382.

Tomazelli, L.J. \& Villwock, J.A. 2000. O Cenozóico no Rio Grande do Sul: geologia da Planície Costeira. In: M. Holz \& L. F. De Ros (eds.) Geologia do Rio Grande do Sul, Edição CIGO/ UFRGS, p. 375-406.
Trejo, V. \& Jackson, D. 1998. Cánidos patagónicos: identificación taxonómica de mandíbulas y molares del sitio arqueológico Cueva Baño Nuevo 1. Anales del Instituto de la Patagonia, Serie Ciencias Humanas, 26:181-194.

Villwock, J.A. \& Tomazelli, L.J. 1995. Geologia costeira do Rio Grande do Sul. Notas Técnicas, 8:1-45.

Webb, S.D. 1985. Late Cenozoic mammal dispersals between the Americas. In: F.G. Stehli, \& S.D.Webb (eds.) The Great American Biotic Interchange, Plenum Press, p. 357-386.

Winge, H. 1895-1896. Jordfunde og nulevende Rovdyr (Carnivora) fra Lagoa Santa, Minas Geraes, Brasilien. E Museo Lundii, 2 (4):1- 103.

Received August, 2003; accepted, December, 2003 